\title{
UV-regularization of field discontinuities
}

\author{
K.Sveshnikov, P.Silaev, and I.Cherednikov \\ Department of Physics and Institute of Theoretical Microphysics, \\ Moscow State University, Moscow 119899, Russia
}

\begin{abstract}
A nonperturbative regularization of UV-divergencies, caused by finite discontinuities in the field configuration, is discussed in the context of 1+1-dimensional kink models. The relationship between this procedure and the appearance of "quantum copies" of classical kink solutions is studied in detail and confirmed by conventional methods of soliton quantization.
\end{abstract}

Particlelike classical solutions (henceforth kinks) and their quantum descendants play a significant role in description of extended objects in the QFT [1-4]. Let $\varphi_{c}(x)$ be the stationary solution of such type, then, as a rule, it interpolates smoothly between static field vacua $\varphi_{v a c}$, corresponding to the global minima of the (effective) energy functional. Now let us note, that with each smooth solution $\varphi_{c}(x)$ one can connect a discontinuous steplike configuration $\varphi_{d}(x)$ with the same topology, whose main difference from $\varphi_{c}(x)$ is the vanishing size of the transition region, where $\varphi \neq \varphi_{v a c}$. For instance, in 1+1-dimensions the familiar kink solution is $\varphi_{c}(x)=\tanh x$, whose discontinuous analog is nothing else, but $\varphi_{d}(x)=\operatorname{sign} x$. The same isituation takes place for more spatial dimensions too, e.g., for skyrmeon-like configurations. Namely, the discontinuous analog for the hedgehog $U(\vec{r})=\exp [i \vec{\sim} \vec{\tau} F(r)]$ can be easily constructed via replacing the radial $\checkmark$ chiral angle function $F(r)$, which varies continuously from $F(0)=\pi n$ to $F(\infty)=0$, by $F_{d}(r)=\pi n \theta(R-r)$ with some finite $R$.

So far, such discontinuous configurations have been dismissed as unimportant physically, on account of two main reasons. The first one is that, purely classically, they do not obey the field equations of motion in the points of discontinuity, where the derivatives of jumps will cause the appearance of singular sources in the r.h.s. The second reason is that at first glance their energy should be infinite due to singularities in the kinetic term. However, in fact the situation turns out to be more subtle, since in the quantum theory certain almost discontinuous configurations of such type appear (at least formally) as proper solutions of field equations in the framework of covariant collective variables [4]. It is worth-while noticing, that in this framework the corresponding modification of field equations originates only from the special operator-valued change of field variables in the model without any changes in the initial Lagrangean. At the same time, the main reason for diverging energy of such discontinuous configurations with finite jumps is that in this case Othe high-frequency component of the field $\tilde{\varphi}(k)$ decreases to slow for $|k| \rightarrow \infty$, and so the abovementioned - divergencies are of an apparent UV-type. However, we know, that at least for some QFT models there exists $\geq$ a reliable way to remove the UV-divergencies via regularization followed by corresponding subtractions. So there appears a natural assumption, that there might exist a reasonable subtracting procedure, which -removes the singularities, caused by such finite jumps. It should be noted, however, that such subtracting should be quite different from the standard renormalization technique. The latter removes the infinities only for perturbations around the ground state, and reveals actually a similar structure in the vacuum as well as in sectors with smooth classical background fields like kinks or solitons [1], whereas in the present case one assumes a nonperturbative procedure, which renormalizes properly the jumps of the field and the divergency in their energy, what obviously cannot be obtained in any finite order of the perturbative expansion.

The purpose of this letter is to present arguments in favour of existence of such nonperturbative subtracting procedure within the context of 1+1-dimensional kink theory, described by the Lagrangean

$$
\mathcal{L}(\varphi)=\frac{1}{2}\left(\partial_{\mu} \varphi\right)^{2}-V(\varphi)
$$

which possesses a stationary kink solution $\varphi_{c}(x)$. Moreover, we'll see, that after such procedure there appears a kind of quantum smoothing of such finite jumps in the sense, that we'll be able to find a series of specific smooth c-number solutions of original field equations, which are of the same topology as the classical kink, but nonanalytically depend on the (effective) Planck constant $\hbar$, i.e., on the factor, that enters the r.h.s. of the CCR $[\varphi(x, t), \pi(y, t)]=i \hbar \delta(x-y)$, and possess the corresponding discontinuous configuration $\varphi_{d}(x)$ as the improper limit for $\hbar \rightarrow 0$.

In implementing this program the crucial point is to quantize the kinks covariantly by means of the relativistic (Newton-Wigner) c.m.s. coordinate [5], which in 1+1-dimensions is given by

$$
q=\frac{1}{2}\left(\frac{1}{H} L+L \frac{1}{H}\right)
$$


where $H, L$ and $P$ are the generators of 1+1-dimensional Poincare algebra

$$
[L, H]=i \hbar P, \quad[L, P]=i \hbar H, \quad[H, P]=0 .
$$

The physical meaning of the operator $q$ implies that it should enter the field arguments only in the combination $(x-q(t))$. It would be convenient to use the following substitution [6-8]

$$
x \rightarrow \xi(x, t)
$$

where

$$
\xi(x, t)=H x-P t-L=H^{1 / 2}(x-q(t)) H^{1 / 2} .
$$

The dimensionless coordinate $\xi(x, t)$, defined in such a way, behaves under the Poincare group transformations $x^{\mu} \rightarrow x^{\prime \mu}=\Lambda_{\nu}^{\mu} x^{\nu}+a^{\mu}$ like a covariant scalar, i.e.

$$
U^{+}(\Lambda, a) \xi(x) U(\Lambda, a)=\xi\left[\Lambda^{-1}(x-a)\right]
$$

provided the generators of unitary transformations $U(\Lambda, a)$ are given by the algebra (3). Through this property any c-number function of it will be a covariant scalar as well. So we are led to represent the Heisenberg field in the kink sector (assuming the validity of weak-coupling expansion in $\sqrt{\hbar}$ for mesonic fluctuations) as follows

$$
\varphi(x, t)=f(\xi(x, t))+\Phi(\xi(x, t), t) .
$$

In eq.(7) $f(\xi(x, t))$ describes the quantum kink, provided $f(\xi)$ is a c-number kink shape function, while $\Phi(\xi(x, t), t)$ denotes the mesonic modes. The latter, considered as a function of the c-number arguments, commutes with the generator of spatial translations $P$, while the commutation relations between $\Phi(\xi, t)$ and other generators of the Poincare group are determined from the covariance properties of the whole field $\varphi(x, t)[6-8]$.

It should be stressed, that eqs.(2-7) are nothing else, but a special change of field variables, which restores the space-time symmetry, broken by the nontrivial c-number solution, directly on the quantum level. The number of degrees of freedom is preserved by equating the Poincare algebra (3) to the corresponding Noether expressions for space-time generators, derived from the initial field Lagrangean (1) $[4,8]$. Moreover, one can show quite unambigously, that it is indeed the repr.(7), that appears as the final result of collecting together all the secular terms in the straightforward perturbation expansion around the stationary classical kink solution [9]. Within expansion in the inverse powers of the kink mass this representation turns out to be equivalent to the canonical methods of soliton quantization, exploring the collective coordinate $X(t)$ [10-12], as well as to quasiclassical expansions, that appear in the BRST-approach [13-15].

At the same time, the appearance of the NW coordinate (2) in field arguments gives rise for some effects of an apparently nonperturbative nature, which take place directly for the kink solutions of the quantized theory. The main circumstance here is, that within the repr.(7) the dynamical eq. of motion for the c-number kink shape $f(\xi)$ acquires a very special form, where all the differential operators are replaced by finite differences with the step $i \hbar$, while the singularities, caused by differentiating of jumps, show up as specific pole terms. These pole terms can be represented in the form, quite analogous to the conventional UV-divergencies of the field, calculated in the rapidity representation. Postulating the subtracting of such pole terms, we'll get a consistent scheme of covariant kink quantization, where the finite jumps of the field are UV-renormalized and instead of divergencies give rise for a series of smooth solutions of field equations, which reproduce the original jump as the improper limit for $\hbar \rightarrow 0$, but their energy remains finite and vanishes, when the size of the solution tends to zero.

To start with, let us insert the repr.(7) into the initial field eq. of motion

$$
\square \varphi+V^{\prime}(\varphi)=0,
$$

with disregard to the mesonic part. Due to the explicit covariance of the kink field $f(\xi(x, t))$ the calculation of space-time derivatives reduces to evaluation of commutators with translation generators

$$
\partial_{\mu} f(\xi(x, t))=\frac{i}{\hbar}\left[P_{\mu}, f(\xi(x, t))\right] .
$$

The calculation of such commutators proceeds as follows. By means of the light-cone operators

$$
K_{ \pm}=H \pm P
$$


one obtains for any integer power of $\xi$

$$
K_{ \pm} \xi^{n}(x, t)=[\xi(x, t) \pm i \hbar]^{n} K_{ \pm}
$$

whence

$$
\square_{x t} \xi^{n}=\left(\frac{i}{\hbar}\right)^{2}\left[K_{+},\left[K_{-}, \xi^{n}\right]\right]=\frac{M^{2}}{\hbar^{2}}\left[(\xi+i \hbar)^{n}+(\xi-i \hbar)^{n}-2 \xi^{n}\right],
$$

where $M^{2}=K_{+} K_{-}=H^{2}-P^{2}$ is the mass operator squared, which commutes with the algebra (3) and so with any $f(\xi(x, t))$. Therefore for any entire function $f(\xi)$ there holds

$$
\square_{x t} f(\xi(x, t))=\sum_{n} f_{n} \square_{x t} \xi^{n}(x, t)=\frac{M^{2}}{\hbar^{2}}[f(\xi+i \hbar)+f(\xi-i \hbar)-2 f(\xi)](x, t),
$$

whence we get the following eq. for the kink shape

$$
f(\xi+i \hbar)+f(\xi-i \hbar)-2 f(\xi)+\frac{\hbar^{2}}{M^{2}} V^{\prime}(f(\xi))=0 .
$$

In eq.(14) the argument $\xi$ is already a c-number, which varies for $-\infty$ to $+\infty$, since the spectrum of the operator $\xi(x, t)$ coincides with that of $(-L)$ and fills in the real axis.

However, the situation changes drastically in the case of $f(\xi)$ being meromorphic. Recalling the Mittag-Leffler theorem [16], we can represent $f(z)$ as a series of its principal parts, which converges in each regular point of $f(z)$ in the complex plane,

$$
f(z)=\sum_{n} \sum_{k=1}^{m_{n}} \frac{c_{-k}^{(n)}}{\left(z-a_{n}\right)^{k}}+\sum_{k=1}^{m} c_{k} z^{k}
$$

thereby it is assumed, that the poles $a_{n}$ don't lye on the lines $\operatorname{Im} z=0, \pm \hbar$. At first glance, the commutation relations between $K_{ \pm}$and inverse powers $\left(\xi-a_{n}\right)^{-k}$ should automatically follow from eq.(11). However, this statement is incorrect, since first of all one has to define selfconsistently what should be meant under $\left(\xi-a_{n}\right)^{-k}$. Namely, the natural definition of any function of the operator $\xi$ should be made via corresponding spectral expansion into the complete set of eigenvectors of $\xi$. Such eigenvectors can be easily found by noticing, that the mass operator $M$ becomes a good quantum number, namely the kink mass, when the mesons are neglected. In this case we can employ the rapidity representation, where the Lorentz boost $L$ takes the form

$$
L=i \hbar \frac{\partial}{\partial \chi}
$$

the canonically conjugated to $L$ variable $\chi$ is the rapidity, while the space-time translation generators are $H=M \cosh \chi, P=M \sinh \chi$. The eigenvectors of $L$ and $\xi=\xi(0,0)=-L$ are given by

$$
\langle\chi \mid L\rangle=(2 \pi \hbar)^{-1 / 2} \exp \left(-\frac{i}{\hbar} L \chi\right),\langle\chi \mid \xi\rangle=(2 \pi \hbar)^{-1 / 2} \exp \left(\frac{i}{\hbar} \xi \chi\right) .
$$

By means of eqs. (16) and (17) one can easily verify, that there hold the following commutation relations between $K_{ \pm}$and $\left(\xi-a_{n}\right)^{-1}$

$$
K_{ \pm} \frac{1}{\xi-a}=\frac{1}{\xi \pm i \hbar-a} K_{ \pm} \pm 2 \pi i K_{ \pm} \delta(\xi-a)
$$

where the term with $\delta$-function appears for $K_{+}$, when $0<\operatorname{Im} a<\hbar$, and for $K_{-}$, when $0>\operatorname{Im} a>-\hbar$, while the $\delta$-function of the complex operator argument is defined through its spectral transform as well. Using eq.(18) and the Mittag-Leffler theorem, we find for any meromorphic $f(\xi)$

$$
K_{ \pm} f(\xi)=f(\xi \pm i \hbar) K_{ \pm} \pm 2 \pi i K_{ \pm} \sum \Gamma_{n} \delta\left(\xi-a_{n}\right)
$$

where $\Gamma_{n}$ are the residues of $f(\xi)$ in the poles $a_{n}$, and to the term with $\delta$-functions contribute only those poles, which lye in the strips $0<\operatorname{Im} a_{n}<\hbar$ for $K_{+}$, and $0>\operatorname{Im} a_{n}>-\hbar$ for $K_{-}$.

From the eq.(19) we immediately find, that whenever the function $f(z)$ possesses the poles in the strip $|\operatorname{Im} z|<\hbar$, the differentiation of the operator $f(\xi(x, t))$ yields besides the regular differences like in eq.(13) 
the additional singular pole terms, containing $\delta\left(\xi-a_{n}\right)$. This are indeed these pole terms, which cause the divergence in the kinetic energy, since the latter is at least quadratic in derivatives and so will contain the integral over $d \xi$ with $\delta$-functions squared. To make this relationship between poles in the strip $|\operatorname{Im} z|<\hbar$ and divergencies in the energy more transparent, let us achieve the same conclusion by considering the matrix elements of the kink operator within the relativized version of the Goldstone-Jackiw approach to soliton quantization [17], when the kink states are taken as the rapidity eigenstates $|\chi\rangle$ with the energy-momentum $\varepsilon=M \cosh \chi, p=M \sinh \chi$.

Following GJ, we define the kink formfactor as

$$
\tilde{f}\left(\chi-\chi^{\prime}\right)=\left\langle\chi|\varphi(0,0)| \chi^{\prime}\right\rangle=\int d \xi\langle\chi \mid \xi\rangle f(\xi)\left\langle\xi \mid \chi^{\prime}\right\rangle=(2 \pi \hbar)^{-1} \int d \xi f(\xi) \exp \left[\frac{i}{\hbar} \xi\left(\chi-\chi^{\prime}\right)\right] .
$$

In terms of the formfactor (20) the matrix element of the Heisenberg kink field can be written as

$$
\left\langle\chi|\varphi(x, t)| \chi^{\prime}\right\rangle=\exp \left\{\frac{i}{\hbar} M\left[t\left(\cosh \chi-\cosh \chi^{\prime}\right)-x\left(\sinh \chi-\sinh \chi^{\prime}\right)\right]\right\} \tilde{f}\left(\chi-\chi^{\prime}\right) .
$$

Taking the matrix element $\left\langle\chi|\ldots| \chi^{\prime}\right\rangle$ from the eq. of motion (8) and using eq.(21), we find in the leading approximation (neglecting mesons)

$$
\frac{2 M^{2}}{\hbar^{2}}\left[\cosh \left(\chi-\chi^{\prime}\right)-1\right]\left\langle\chi|\varphi(x, t)| \chi^{\prime}\right\rangle+\langle\chi| V^{\prime}\left(\varphi(x, t)\left|\chi^{\prime}\right\rangle=0\right.
$$

whence by removing the $x t$-dependence and going over to $f(\xi)$ by means of eq.(20) we'll get nothing else but the finite-difference eq.(14), provided $f(z)$ is analytic in the strip $|\operatorname{Im} z|<\hbar$. The last requirement is crucial for the equivalence between eqs.(14) and (22), since otherwise the Fouriet-transform of the kinetic term in eq.(22) doesn't exist due to the additional exponential factor $\cosh \left(\chi-\chi^{\prime}\right)$.

The same picture we'll find by evaluating the kink energy in the GJ-approach. Due to the explicit covariance it suffers to calculate only the kink mass for the states at rest, which is given by the normalized matrix element

$$
M[f]=\left.\frac{\left\langle\chi^{\prime}|H(\varphi)| \chi\right\rangle}{\left\langle\chi^{\prime} \mid \chi\right\rangle}\right|_{\chi=0}
$$

where $H(\varphi)$ is the field Hamiltonian

$$
H(\varphi)=\int d x\left[\frac{1}{2}\left(\partial_{t} \varphi\right)^{2}+\frac{1}{2}\left(\partial_{x} \varphi\right)^{2}+V(\varphi)\right]
$$

Removing the spatial integration, we get

$$
M=\left.2 \pi \hbar \delta\left(M \sinh \chi-M \sinh \chi^{\prime}\right) \frac{\left\langle\chi^{\prime}|\mathcal{H}(0)| \chi\right\rangle}{\left\langle\chi^{\prime} \mid \chi\right\rangle}\right|_{\chi=0}=\left.\frac{2 \pi \hbar}{M \cosh \chi}\langle\chi|\mathcal{H}(0)| \chi\rangle\right|_{\chi=0}
$$

where $\mathcal{H}(x)$ is the Hamiltonian density. In the last expression the main attention should be paid to the kinetic term with the field derivatives

$$
T=\frac{1}{2}\left[\left(\partial_{t} \varphi\right)^{2}+\left(\partial_{x} \varphi\right)^{2}\right] .
$$

Following GJ, by evaluating $\langle\chi|T| \chi\rangle$ we insert into the matrix element the intermediate set of the kink states $\int d \chi^{\prime}\left|\chi^{\prime}\right\rangle\left\langle\chi^{\prime}\right|$ and find

$$
\langle\chi|T| \chi\rangle=\frac{M^{2}}{2 \hbar^{2}} \int d \chi^{\prime}\left[\left(\cosh \chi-\cosh \chi^{\prime}\right)^{2}+\left(\sinh \chi-\sinh \chi^{\prime}\right)^{2}\right]\left|\tilde{f}\left(\chi-\chi^{\prime}\right)\right|^{2} .
$$

Now, if we put $\chi=0$ and replace formally $\tilde{f}(\chi)$ by $f(\xi)$, we'll get the following expression

$$
\left.\langle\chi|T| \chi\rangle\right|_{\chi=0}=(2 \pi \hbar)^{-1} \frac{M^{2}}{2 \hbar^{2}} \int d \xi\left\{\left[\left(\cos \hbar \partial_{\xi}-1\right) f(\xi)\right]^{2}+\left[\left(\sin \hbar \partial_{\xi}\right) f(\xi)\right]^{2}\right\} .
$$

However, in general such transition from eq.(27) to (28) is incorrect, since due to the exponential factor $\left[\left(\cosh \chi-\cosh \chi^{\prime}\right)^{2}+\left(\sinh \chi-\sinh \chi^{\prime}\right)^{2}\right]$ the expression $(27)$ diverges for such $f(\xi)$, for which (28) remains 
well-defined. In particular, it takes place when $\tilde{f}(\chi)$ decreases for $|\chi| \rightarrow \infty$ slowlier than $\exp (-|\chi|)$, what is just the case when the pole of $f(z)$ appears in the strip $|\operatorname{Im} z|<\hbar$. It is indeed this circumstance, that makes the interchange of integrations by calculation of the matrix element $\langle\chi|T| \chi\rangle$ in general illegal and so yields an infinite difference between eqs.(27) and (28). This difference is nothing else, but the quantum descendant of the classical singularity, caused by the jump in the field configuration, since in the quasiclassical limit $\hbar \rightarrow 0$ the existence of the pole in the strip $|\operatorname{Im} z|<\hbar$ implies, that it approaches the real axis, creating the discontinuity in $f(\xi)$. At the same time, the covariant approach to kink quantization, described above, permits us to separate by means of the eq.(19) the corresponding singularities in the field derivatives as the additional pole terms, containing $\delta$-functions of the complex argument. Now let us present some arguments (without any claim of generality), that by quantization the field in the rapidity (or $\xi \chi^{-}$) representation instead of the conventional $x p$-one, such $\delta$-functions turn out to be the natural form of representing the UV-singularities of the theory. For convenience we put the mass of the elementary quantum of the theory equal to unity $m=1$, but retain the $\hbar$-dependence.

In the standard $x p$-representation the canonical field variables are

$$
\varphi(x)=\frac{1}{\sqrt{2 \pi \hbar}} \int \frac{d p}{\sqrt{2 \omega_{p}}}\left[a_{p} e^{i p x / \hbar}+\text { h.c. }\right], \quad \pi(x)=\frac{i}{\sqrt{2 \pi \hbar}} \int \sqrt{\frac{\omega_{p}}{2}} d p\left[a_{p}^{+} e^{-i p x / \hbar}-h . c .\right]
$$

where

$$
\left[a_{p}, a_{p^{\prime}}^{+}\right]=\hbar \delta_{p p^{\prime}}, \quad\left[a_{p}, a_{p^{\prime}}\right]=0 .
$$

The rapidity representation $p=\sinh \chi, \omega_{p}=\cosh \chi$ is introduced by the following canonical change of creation-annihilation operators

$$
a_{p}=a_{\chi} / \sqrt{\cosh \chi}
$$

provided

$$
\left[a_{\chi}, a_{\chi^{\prime}}^{+}\right]=\hbar \delta_{\chi \chi^{\prime}}, \quad\left[a_{\chi}, a_{\chi^{\prime}}\right]=0
$$

The appropriate field variables for $\xi \chi$-representation are

$$
\Phi(\xi)=\frac{1}{\sqrt{2 \pi \hbar}} \int \frac{d \chi}{\sqrt{2}}\left[a_{\chi} e^{i \chi \xi / \hbar}+h . c .\right], \quad \Pi(\xi)=\frac{i}{\sqrt{2 \pi \hbar}} \int \cosh \chi \frac{d \chi}{\sqrt{2}}\left[a_{\chi}^{+} e^{-i \chi \xi / \hbar}-h . c .\right],
$$

and obey the following commutation relations

$$
\left[\Phi(\xi), \Pi\left(\xi^{\prime}\right)\right]=\frac{i}{2 \pi} \int d \chi \cosh \chi e^{i \chi\left(\xi-\xi^{\prime}\right) / \hbar}=\frac{i \hbar}{2}\left[\delta\left(\xi-\xi^{\prime}+i \hbar\right)+\delta\left(\xi-\xi^{\prime}-i \hbar\right)\right] .
$$

So the rapidity repr. contains from the beginning the $\delta$-functions of the complex argument, quite similar to those of eq.(19). On the other hand, it is indeed this singularity in the field commutator (34), which causes ultimately all the UV-divergencies of the quantum theory. So it should be clear, that in the rapidity representation all the divergent quantities can be written in the same fashion. For instance, for the vacuum energy of the free field one has

$$
E_{v a c}=\frac{1}{2} \int \omega_{p} d p\left(a_{p} a_{p}^{+}-a_{p}^{+} a_{p}\right)=\frac{1}{2} \int \cosh \chi d \chi\left(a_{\chi} a_{\chi}^{+}-a_{\chi}^{+} a_{\chi}\right),
$$

what can be expressed through the field commutator as

$$
E_{v a c}=\frac{1}{2 i} \int d \xi d \xi^{\prime} \delta\left(\xi-\xi^{\prime}\right)\left[\Phi(\xi), \Pi\left(\xi^{\prime}\right)\right]=\frac{\hbar}{2} \int d \xi d \xi^{\prime} \delta\left(\xi-\xi^{\prime}\right) \frac{1}{2}\left[\delta\left(\xi-\xi^{\prime}+i \hbar\right)+\delta\left(\xi-\xi^{\prime}-i \hbar\right)\right] .
$$

Of course, the eq.(36) as well as (35) give the same final divergent answer $E_{v a c}=(\hbar / 2) \int \cosh \chi d \chi$. However, the main result of this exercise is that the rapidity repr. shows up a direct relationship between UVdivergencies and $\delta$-functions of the complex argument.

Motivated by these arguments, we assume now, that such $\delta$-functions should be subtracted whenever they appear in calculations. For the perturbation theory this assumption leads to the conventional renormalization techniques, whereas in the present case it implies the subtraction of the pole terms from eq.(19). Such procedure regularizes the field derivatives for finite jumps and so is equivalent to an effective UV-cutoff, implemented in a nonperturbative way. Note, that this subtraction cannot be understood as a result of partial resummation of the regularized perturbation series, since here we are strictly related to the knowledge of the poles and residues $a_{n}$ and $\Gamma_{n}$ of the given solution $f(\xi)$, and so are not able (or al least 
do not know how) to reformulate such procedure as adding a finite number of universal counterterms to the initial Lagrangean, what is the charge for nonperturbativity. Moreover, even for a renormalizable theory the perturbation series might be divergent. So our approach should be merely understood as an attempt to give sense to the theory in the case, when the conventional methods of UV-regularization do not work.

Removing the pole terms from field derivatives, we get the finite-difference eq.(14) as the dynamical eq. for the kink shape $f(\xi)$. However, now $f(z)$ is allowed to possess poles in the strip $|\operatorname{Im} z|<\hbar$, it should be regular only on the lines $\operatorname{Im} z=0, \pm \hbar$. Proceeding further, we'll find, that the equation for the kink mass will also contain the same finite-difference structure. Namely, expressing the field derivatives as commutators and subtracting the pole terms, we represent the kinetic energy as

$$
\begin{gathered}
T=\frac{1}{2}\left[\left(\partial_{t} \varphi\right)^{2}+\left(\partial_{x} \varphi\right)^{2}\right]=\frac{i^{2}}{4 \hbar^{2}} \sum_{ \pm}\left\{K_{ \pm}[f(\xi)-f(\xi \mp i \hbar)][f(\xi \pm i \hbar)-f(\xi)] K_{ \pm}\right\}= \\
=\frac{i^{2}}{2 \hbar^{2}}\{H[f(\xi)-f(\xi-i \hbar)][f(\xi+i \hbar)-f(\xi)] H+P[f(\xi)-f(\xi-i \hbar)][f(\xi+i \hbar)-f(\xi)] P\},
\end{gathered}
$$

with the same minimal conditions on $f(\xi)$. The kink mass is defined as the matrix element (23), and to calculate $\langle\chi|\mathcal{H}(0)| \chi\rangle$ we insert the complete set $\int d \xi|\xi\rangle\langle\xi|$. After some simple algebra the final answer is

$$
2=\frac{2}{2 \hbar^{2}} \int d \xi[f(\xi+i \hbar)-f(\xi)][f(\xi-i \hbar)-f(\xi)]+\int d \xi V(f(\xi)) .
$$

The kinetic term here coincides completely with the "regularized" version (28) of $\langle\chi|T| \chi\rangle$, computed by the GJ-approach.

Now let us turn to the kink content of the theory after such regularization of finite jumps. First of all, the smooth solutions of the theory remain unchanged, since by definition they should be analytic in the strip $|\operatorname{Im} z|<\hbar \mid$, so there are no subtractions at all. The most convenient way to see this is to expand the eq.(14) in powers of $\hbar$. Then to the leading order we get

$$
-M_{0}^{2} f^{\prime \prime}(\xi)+V^{\prime}(f)=0,
$$

where $M_{0}$ is the quasiclassical ( $\hbar \rightarrow 0$ ) limit of the quantum kink mass $M$. The eq.(39) is nothing else, but the classical stationary kink equation of motion, provided $x=\xi / M_{0}$. So if the proper limit $\hbar \rightarrow 0$ for $f(\xi)$ exists, it should be equal to

$$
f(\xi)=\varphi_{c}\left(\xi / M_{0}\right)
$$

where $\varphi_{c}(x)$ is the classical kink solution. Performing a similar expansion for the mass eq.(38), we'll find

$$
M_{0}^{2}=\int d \xi\left[\frac{M_{0}^{2}}{2} f^{\prime}(\xi)^{2}+V(f(\xi))\right],
$$

whence by means of eq.(40) we immediately obtain for $M_{0}$ the classical expression for the kink mass

$$
M_{0}=\int d x\left[\frac{1}{2} \varphi_{c}^{\prime}(x)^{2}+V\left(\varphi_{c}(x)\right)\right]
$$

However, in contrast to the classical eq.(39), the finite-difference eq.(14) admits for a set of other kink solutions, which are in some sense generated by the classical one, but are irregular for $\hbar \rightarrow 0$, and so should be called its "quantum copies". The derivation of such solutions has been discussed in detail in ref. [4]. The result is

$$
f_{N}(\xi)=\varphi_{c}\left(\alpha_{N} \xi\right)
$$

where $N$ is an integer,

$$
\alpha_{N}=\frac{1}{M_{N}}+\frac{2 \pi N}{m \hbar}
$$

with $M_{N}=M\left[f_{N}\right]$ being the corresponding mass, while $m$ is the mass of the elementary quantum (meson). The solutions (43) are exact up to $O(\hbar)$, the first correction to them appears at the one-loop level and so can be freely ignored to the leading order. For $N=0$ the eq.(43) coincides with (40), and this is the only case, when the solution is regular for $\hbar \rightarrow 0$. For $N \neq 0$ we get for $M_{N}$

$$
M_{N}=\sqrt{\frac{M_{0}}{\left|\alpha_{N}\right|}} \simeq \sqrt{\frac{\hbar}{|N|}}\left(\frac{M_{0} m}{2 \pi}\right)^{1 / 2} .
$$


Therefore the masses of quantum kink copies are proportional to $\sqrt{\hbar}$ and so lye in between the classical kink mass, which is $O(1)$, and meson energies, whose magnitude is $O(\hbar)$. Moreover, $M_{N}$ decreases for increasing $|N|$. A similar behaviour reveals the size of solutions (43), which turns out to be $O(\hbar /|N|)$. So for $\hbar / N \rightarrow 0$ the size of the copy vanishes, and the copy shrinks into an almost discontinuous steplike configuration $f_{\infty}(\xi)$ of the same topology, as the initial classical kink. In other words, we rediscover the discontinuous steplike classical solution $\varphi_{d}(x)$, introduced at the beginning, as the (improper) limit of quantum copies for $\hbar / N \rightarrow 0$

$$
f_{N}(\xi) \rightarrow f_{\infty}(\xi)=\varphi_{d}(\xi)
$$

According to eq.(45) the mass of $f_{\infty}(\xi)$ vanishes. This result is a direct consequence of that through our subtracting procedure we have introduced an effective UV-cutoff, because when the size of the copy decreases, the relative weight of the high-frequency component of the field increases and so yields the main (divergent) contribution to all the field observables, what is exactly cancelled by the proposed subtraction. Mathematically this result is trivial, since $f_{\infty}(\xi)$ is purely discontinuous, and so its derivatives contain only $\delta$-functions, after subtraction of which the energy vanishes exactly. At the same time, without subtraction of the pole terms none of copies is possible, at least for 1+1-dimensional kink models. This happens because in this case $\varphi_{c}(x)$ can be represented as a (Dirichlet) series in powers of $e^{ \pm m x}$ (depending on the sign of $x$ ), so is $2 \pi i / m$ periodic. On the other hand, for the Nth copy the strip $|\operatorname{Im} z|<\hbar$ maps into $|\operatorname{Im} z|<2 \pi N / m$ for $\varphi_{c}(z)$, where it must be analytic for the given copy to exist without subtraction. So $\varphi_{c}(z)$ must be an entire function, but this is not the case for the kink models of interest.

It should be noted, however, that the relation (46), being quite rigorous in the mathematical sense, cannot take place physically. First of all, for vanishing energy the NW c.m.s. coordinate (2) as well as the substitution (4) become ill-defined. Secondly, in our approach the appearance of the kink (or its copy) is considered as the main dynamical effect, and quantum fluctuations of the field are treated only perturbatively, what might be correct only until the mass of the kink is sufficiently larger than the mesonic energies $\hbar \omega_{k}$. Otherwise one has to consider the mesonic excitations at the same footing with the kink, what might significantly disturb the whole dynamical picture. From this point of view the masses of copies should be bounded from below by $O(\hbar)$, and so there appears an upper bound $|N|_{\max } \simeq O(1 / \hbar)$, what corresponds to the copy with the minimal mass and size, allowed for given $\hbar$. With disregard to quantum corrections the latter looks like the limiting $f_{\infty}(\xi)$, but the abovementioned difference between them is principal, since it shows that the formal appearance of $f_{\infty}(\xi)$ with zero mass doesn't actually contradict the uniqueness of the vacuum state.

Let us also mention, that the appearance of the kink copies is a highly relativisitic effect. If we restore the speed of light explicitly, we'll find

$$
[L, P]=i \hbar \frac{H}{c^{2}}
$$

what scales the step in finite differences up to $i \hbar / c$. Therefore there are in fact two independent parameters in our approach, the first one is $\hbar^{\prime}=\hbar / c$ and describes the finite-difference effects, while the second $-\sqrt{\hbar}$ - serves as the expansion parameter for dealing with mesonic fluctuations.

To be more concrete, let us consider as an illustrative example the standard $\varphi^{4}$-model, when the selfinteraction of the field is

$$
V(\varphi)=\frac{1}{2}\left(1-\varphi^{2}\right)^{2}
$$

and yields the classical kink solution

$$
\varphi_{c}(x)=\tanh x
$$

Its copies are

$$
f_{N}(\xi)=\tanh \alpha_{N} \xi
$$

where $\alpha_{N}=1 / M_{N}+\pi N / \hbar$, since now $m=2$. The functions (50) are meromorphic with simple poles at $a_{n}=i \pi(n+1 / 2) / \alpha_{N}$, so there are exactly $2 N$ poles in the strip $|\operatorname{Im} z|<\hbar$. The kink copies (50) do not be the exact solutions of eq.(14), but satisfy the eq.

$$
\square f_{N}+2 \frac{\tan ^{2} \beta}{\beta^{2}} \frac{f_{N}\left(f_{N}^{2}-1\right)}{1+f_{N}^{2} \tan ^{2} \beta}=0,
$$

where $\beta=\hbar / M_{N}$ and so is $O(\sqrt{\hbar})$. Because $\left|f_{N}(\xi)\right| \leq 1$ for all real $\xi$, the difference between the "potential" term in eq. (50) and the correct one $2 f_{N}\left(f_{N}^{2}-1\right)$ turns out to be $O(\hbar)$ on the whole $\xi$-axis, as it was stated above. 
The topology of kinks (50) is the same as of the classical one, so they carry the same topological charge $Q= \pm 1$ and shrink for $\hbar / N \rightarrow 0$ into the discontinuous sign solution with vanishing mass. For the kinks (50) the last effect can be made even more apparent, since for $\hbar / N$ sufficiently small they can be approximated by $i \hbar$-periodic functions tanh $(\pi N \xi / \hbar)$, obeying $f(\xi \pm i \hbar)=f(\xi)$. For such functions the kinetic term in the mass eq.(38) will vanish identically, while the potential term is nonzero only in the transition region, where $|f(\xi)| \neq 1$, and so vanishes for $\hbar / N \rightarrow 0$.

To conclude we have shown, that the finite jumps of the field profile might be regularized in a way, being equivalent to introducing an effective UV-cutoff and respecting the Lorentz-covariance from the very beginning. As a result, the energy (mass) of the discontinuous steplike solution, defined as the (improper) limit of copies (43), becomes vanishing, rather than diverging. A number of accompanying effects, the most important of which is the possibility of the kink collapse into its copy, is discussed elsewhere [18].

This work has been done by partial financial support of RFFI, grant No.96-02-18097, S-P Concurrency Center of Fundamental Research, grant No. 95-0-6.3-20 and the Scientific Program "Russian Universities".

\section{References}

[1] R.Rajaraman, Solitons and Instantons (North-Holland, Amsterdam, 1982).

[2] L.Wilets, Nontopological Solitons (World Scientific, Singapoure, 1989).

[3] V.G.Makhan'kov, Y.P.Rybakov, V.I.Sanyuk, The Skyrme Model, Springer Series in Nuclear and Particle Physics (Springer, Berlin, 1993).

[4] K.Sveshnikov, Nucl.Phys. B405 (1993) 451; Int.Journ.Mod.Phys. A9 (1994) 2586.

[5] T.D.Newton and E.P.Wigner, Rev.Mod.Phys. 21 (1949) 400.

[6] H.Matsumoto, N.J.Papastamatiou, H.Umezawa, and M.Umezawa, Phys. Rev. D23 (1981) 1339.

[7] H.Matsumoto, N.J.Papastamatiou, G.Semenoff, and H.Umezawa, Phys. Rev. D24 (1981) 406.

[8] K.Sveshnikov, Teor.Mat.Fiz. 55 (1983) 361; 74 (1988) 373.

[9] G.Semenoff, H.Matsumoto, and H.Umezawa, Journ.Math.Phys. 22 (1981) 2208; Progr.Theor.Phys. 67 (1982) 1619.

[10] J.-L.Gervais, A.Jevicki, and B.Sakita, Phys.Rev. D12 (1975) 1038.

[11] E.Tomboulis, Phys.Rev. D12 (1975) 1678.

[12] N.-H. Christ and T.D.Lee, Phys.Rev. D12 (1975) 1606.

[13] A.Calogeracos, Phys.Rev. D42 (1990) 573.

[14] F.Aldabe, D.R.Bess, N.N.Scoccola, Phys.Lett. B293 (1992) 81; B304 (1993) 98.

[15] J.P.Garrahan, M. Kruczenski, G.L. Schat, D.R.Bess, N.N.Scoccola, Phys. Rev. D51 (1995) 2950.

[16] M.A.Lavrent'ev, B.V.Shabat, Methods of the theory of functions of the complex variable, (State Publishing House of Physical \& Mathematical Literature, Moscow. 1958).

[17] J.Goldstone, R.Jackiw, Phys.Rev. D11 (1975) 1486.

[18] K.Sveshnikov, Mod.Phys.Lett. A10 (1995) 1101; K.Sveshnikov and P.Silaev, Teor.Mat.Fiz., in print. 